\title{
Damage and Permeability in Quasi-brittle Materials: from Diffuse to Localized Properties
}

Gilles PIJAUDIER-CABOT, Frédéric DUFOUR and Marta CHOINSKA.

\subsection{Introduction}

Transport properties of concrete, like permeability or diffusivity, are particularly important in the case of structures for which tightness is important, for instance for pre-stressed concrete containment vessels in nuclear power plants. For such sensitive nuclear vessels, tightness to gas is critical during their service life, where concrete remains at most micro-cracked, but also upon minor accidents, when macro-cracks may appear locally. Thus, it is important to provide relationships between the amount of cracking and damage in concrete and its intrinsic permeability.

Experimental test data (Choinska et al. 2007) performed on hollow concrete cylinders subjected to compressive loading and at the same time gas flow through their thickness exhibit three regimes of growth of permeability. In a simple compression test, the first regime ranges almost up to the peak stress. It exhibits a slow increase of permeability due to the increase of the density of diffuse microcracks. Due to strain localization (transition between diffuse micro-cracking and macro-crack formation), a second regime of permeability growth with a fast increase is observed experimentally, close to the peak stress/peak strain. Finally, and for an applied strain which is larger than several times the peak strain, a third regime is reached. It is characterized by a slower rate of growth of permeability that is 
consistent with Poiseuille flow as we will see further. Figure 15.1 shows these regimes as measured by Choinska et al. (2007) in compression.

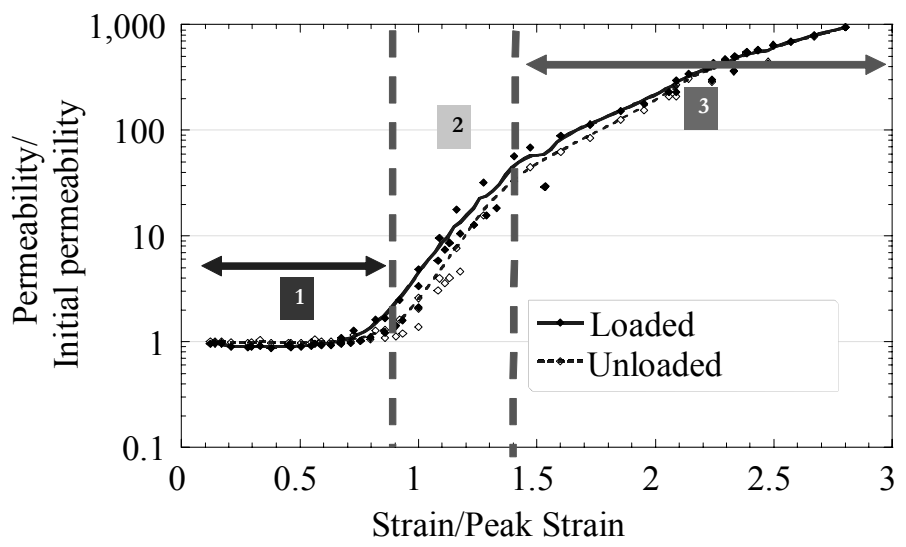

Figure 15.1. Evolution of the permeability as a function of the applied strain in a compression test on a hollow cylinder with a radial gas flow

Relationships between permeability and diffuse micro-cracking, described by a damage variable, have already been derived theoretically (Dormieux and Kondo, 2004 and Chatzigeorgiou et al. 2005) and investigated experimentally (Picandet et al. 2001). This case corresponds to the first regime of the above figure. When macro-cracks have completely formed, it seems consistent to assume that the apparent permeability of the specimen should be governed by the crack opening. We expect that this case corresponds to regime 3 in Figure 15.1.

Between these two extreme cases, there is a transition regime that needs to be properly captured. This is the purpose of the present contribution, where we introduce a law governing the growth of intrinsic permeability of a quasi-brittle material (concrete) as a function of damage. In doing so, we are going to define a consistent matching relationship between the two above extreme regimes.

The equations that describe the mechanical problem are briefly recalled in section 15.2. The basic reasoning for establishing the matching law is provided in section 15.3. Section 15.4 is devoted to the calculation of the estimate of the crack opening needed in the localized damage regime. Finally, section 15.5 discusses numerical results and comparisons with existing test data in the literature. 


\subsection{Mechanical problem - continuum damage modeling}

Fracture and damage mechanics are two correlated theories (Mazars and Pijaudier-Cabot 1996). In a computational setting, it is still more convenient to deal with continuum damage instead of having to describe the shape, length and orientation of cracks (including their interaction). For this reason, we are going to use a continuum damage approach. The scalar damage model developed by Mazars (1984) enriched by a non-local approach to strain softening has been selected because it offers a robust and quite simple set of constitutive relations. The same developments could be performed on the basis of a more complex description of damage, anisotropic damage, without major difficulties. It would provide some information about the anisotropy of the permeability that is embedded into the description of the Poiseuille flow between the two faces of a macro-crack. Here, directionality of damage and permeability are not considered for simplicity, but the permeability corresponds to fluid flow parallel to possible (micro) crack faces, in a plane normal to the applied tensile stresses.

Damage is assumed to be isotropic and it produces a degradation of the elastic stiffness of the material through a variation of the Young's modulus:

$$
\sigma=(1-D) C \varepsilon
$$

where $\sigma$ and $\varepsilon$ are the Cauchy stress tensor and the strain tensor respectively. $C$ is the fourth order tensor of elastic moduli. The damage variable $D$ ranges from 0 for virgin material to 1 for completely damaged material with a zero stiffness and depends on state variable $Y$ :

$$
D=F(Y)
$$

The state variable $Y$ reaches a maximal value during loading history between the damage threshold $Y_{D 0}$ and the equivalent strain $\varepsilon_{e q}$ :

$$
Y=\max _{/ t}\left(\varepsilon_{e q}, Y_{D 0}\right)
$$

The equivalent strain $\varepsilon_{e q}$ is defined as follows (Mazars 1984):

$$
\varepsilon_{e q}=\sqrt{\sum_{i=1}^{3}\left(\left\langle\varepsilon_{i}\right\rangle_{+}\right)^{2}}
$$


Damage follows a damage evolution law which distinguishes tensile damage $D_{t}$ and compressive damage $D_{c}$ (Mazars 1984):

$$
D=\alpha_{t} D_{t}+\alpha_{c} D_{c}
$$

where $\alpha_{t}$ and $\alpha_{c}$ are the weights calculated from the strain tensor. In the present case, only damage due to tension is considered and $\alpha_{t}=1$ and $\alpha_{c}=0$. Therefore, the damage model used hereafter is based on the following evolution damage law:

$$
D=D_{t}=1-\frac{Y_{D 0}\left(1-A_{t}\right)}{Y}-\frac{A_{t}}{e^{\left[B_{t}\left(Y-Y_{D 0}\right)\right]}}
$$

where $A_{t}$ and $B_{t}$ are the model parameters. In this formulation, damage is defined locally. This local formulation exhibits spurious strain localization (like any other strain softening local formulation). Consequently, numerical simulations yield a pathological mesh dependency and physically unrealistic results are obtained (Bazant and Planas 1998).

One possible remedy consists of reformulating the constitutive model in a nonlocal approach, with damage at each material point depending on the strain not only at this point, but also in its neighborhood. This non-local model was developed by Pijaudier-Cabot and Bazant (1987). In this model, a non-local equivalent strain is the weighted average of the local strains over the representative volume $\Omega$ surrounding each point $x$ in the material:

$$
\bar{\varepsilon}_{e q}(x)=\frac{\int_{\Omega} \phi(x-s) \varepsilon_{e q}(s) d \Omega}{\int_{\Omega} \phi(x-s) d \Omega}
$$

$\phi(x-s)$ is the usual weight function defined as:

$$
\phi(x-s)=e^{-\left(\frac{2\|x-s\|}{l_{c}}\right)_{2}}
$$

$l_{c}$, the internal length of the material, is related to the material heterogenity parameter quantifying the non-local interactions. 


\subsection{Permeability matching law}

We are going to focus on the intrinsic permeability of the material free of any stress and we will disregard the reversible stress effect on the permeability. Before describing how the permeability can be defined over the entire range of variation of damage, let us first discuss the two extreme cases of diffuse and localized damage.

\subsubsection{Diffuse damage}

In order to represent the interaction between diffuse damage and permeability at material level, the phenomenological relation established by Picandet et al. (2001) for damage lower than 0.15 is retained. The intrinsic material permeability of the stress free material is an exponential function of damage:

$$
k_{D}=k_{0} \exp \left[(\alpha D)^{\beta}\right]
$$

where $k_{D}$ and $k_{0}$ are respectively the current and the initial material permeability. $\alpha$ and $\beta$ are the parameters fitted by the author to 11.3 and 1.64 respectively. This relationship has been fitted in the case of compression damage. It is assumed that it holds similarly in the case of tension damage too. As reported by Picandet and coworkers, equation [15.9] with the reported values of the material parameters holds for a variety of concretes (ordinary, high performance, fiber reinforced) and it has been selected so that for standard concrete mixes, it can be implemented without any determination of material parameters, as a first approximation.

\subsubsection{Localized damage - crack opening versus permeability}

At complete rupture, a macro-crack (or several macro-cracks) is expected. Hence, fluid flow will be governed by these cracks and Poiseuille's law may be considered. For a fluid flowing between two parallel (rough) plates, the permeability is provided by the expression:

$$
k_{p}=\zeta \frac{[u]^{2}}{12}
$$

$[u]$ is the crack opening and $\zeta$ is the roughness of the crack. Accordingly, and if we consider a crack of length $L$ in a specimen of cross-section $S$ exposed to fluid flow, the total flux per unit thickness of specimen provides by adding the fluid flow through the crack and the fluid flow through the undamaged material around the crack, the apparent permeability $k_{a p}$ : 


$$
k_{a p} S=\zeta \frac{[u]^{2}}{12} \cdot[u] L+k_{0} S
$$

Outside the crack, the permeability is assumed to remain equal to the initial one. It is as if we were looking at the material permeability outside the fracture process zone and it is also a standard assumption in double porosity models. The "crack permeability", called $k_{f}$, is:

$$
k_{f}=\zeta \frac{[u]^{3}}{12} \cdot \frac{L}{S}
$$

This permeability is the second asymptotic case. It is the material permeability that should be reached when damage is close to 1 , at material failure. The difficulty is that it is a geometry dependent parameter. It contains the crack length and the cross-section of the material exposed to fluid flow. Within a continuum description of damage, the above expression of the permeability requires the knowledge of the crack location, its length and the possible crack spacing in addition to the crack opening. A more convenient formulation of the contribution of the crack to the apparent permeability can be derived assuming that the crack is replaced by a band of intense damage.

Let us call $\lambda l_{c}$ the width of this band. It is proportional to the internal length since the width of the fracture process zone in a non-local damage model is indeed a linear function of the internal length. The apparent permeability now derives from the flux in the band with material permeability $k_{l}$ (where damage is assumed to be constant for simplicity):

$$
k_{a p} \cdot S=\lambda l_{c} k_{l} \cdot L+k_{0} S
$$

We may now equate equations [15.11] and [15.13] and the material permeability $k_{l}$ is:

$$
k_{l}=\frac{\zeta[u]^{3}}{12 \lambda l_{c}}
$$

The local - point-wise - permeability in equation [15.14] does not depend on the geometry of the considered specimen. It may readily be used in a continuum model. If we consider a crack of length $L$ in a specimen of cross-section $S$ exposed to fluid flow, equation [15.13] will be recovered at the structural level (apparent average 
permeability). The remaining difficulty is the calculation of the crack opening. This point will be discussed in section 15.4.

\subsubsection{Matching law}

As two permeability evolution laws, $k_{D}$ (equation [15.9]) and $k_{l}$ (equation [15.14]), have already been formulated using the damage variable only, we associate them by means of a simple matching law based on logarithm of the permeability:

$$
\log (k)=(1-D) \log \left(k_{D}\right)+D \log \left(k_{l}\right)
$$

In addition, there is an obstacle concerning application of Picandet's exponential relation in the proposed matching law. Picandet's relation, valid for diffuse and moderate damage ranging between 0 and 0.15 , quickly tends towards infinite values when damage increases. Thus, we use the Taylor expansion of Picandet's relation for small damage in order to avoid a spurious exponential increase of permeability for high values of damage:

$$
k_{D}{ }^{P}=k_{0}\left[1+(\alpha D)^{\beta}+\frac{(\alpha D)^{2 \beta}}{2}+\frac{(\alpha D)^{3 \beta}}{6}\right]
$$

\subsection{Calculation of a crack opening in continuum damage calculations}

The derivation of a crack opening as a result of a continuum damage mechanics calculation is a major challenge. In fact, once a continuum damage model has been selected in order to model discontinuous failure, it is not easy to proceed in the reverse direction. According to damage modeling, there is no such thing as a displacement discontinuity. In most recent papers, this problem is tackled by the assumption that above a given critical value of damage, a displacement discontinuity exists and it should be incorporated in the structural model. From this starting point onwards, advanced computational models e.g. based on X-FEM kinematics provide sound techniques for calculating a displacement discontinuity. 


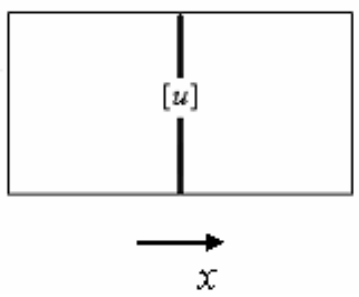

(a)

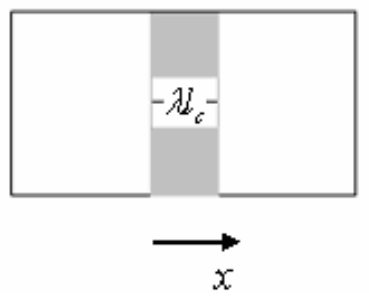

(b)

Figure 15.2. (a) Cracked domain (discrete case); (b) damaged domain (continuous case)

In a more recent proposal, Dufour et al. (2008) have devised a technique for extracting a discontinuity of displacement from a continuum damage FE calculation without the need for enhancing the kinematics with displacement discontinuities. This technique also provides an indicator of the quality of the displacement discontinuity estimate. It should be possible to implement the above techniques in order to calculate the displacement discontinuity at each point of the crack (intense damage zone), but in a first step, we shall devise a simpler and more straightforward approximation of the displacement jump $[u]$.

In order to calculate a crack opening from a damage field, we assume again that the crack, according to the damage model, is described as a band of intense damage of width $\lambda l_{c}$. An equivalence between two domains is then considered: a cracked one with a discontinuous crack opening $[u]$ (see Figure 15.2(a)) and a damaged one with a damaged zone of width $\lambda l_{c}$ (see Figure 15.2(b)). This equivalence states that the displacement across the crack is described as the integral of the strain across the damage band. Subsequently, we assume that the loading is monotonically increasing and we relate the strain inside the band to the variable that controls damage equation [15.3]. In the present case and according to Mazars' model, the two quantities are similar and the crack opening is now expressed as:

$$
[u]=\int_{0}^{\lambda l_{c}}\left(\bar{Y}-Y_{D 0}\right) d x
$$

Assuming that the damage distribution in the damaged zone is uniform, we obtain the crack opening as:

$$
[u]=\left(\bar{Y}-Y_{D 0}\right) \lambda l_{c}
$$


To substitute this crack opening with a damage field, we relate the state variable with the damage using the inverse of the damage evolution law (see equation $[15.2])$ :

$$
\bar{Y}=F^{-1}(D)
$$

Finally, by substitution of equation [15.19] in equation [15.18], the crack opening may be represented as a function of the damage:

$$
[u]=\left(F^{-1}(D)-Y_{D 0}\right) \lambda l_{c}
$$

This equation may now be substituted in equation [15.14], and further in equation [15.15] in order to assign local values of the permeability point-wise. It should be recalled that this derivation remains based on simple - not to say simplistic - assumptions. Again, it may be improved by considering variation of the state variable in the damage band or by application of the method presented by Dufour et al. (2008).

For the sake of illustration, let us consider the material parameters given in Table 15.1 and let us calculate the evolution of material permeability as described by the matching law in equation [15.15].

\begin{tabular}{|l|l|}
\hline$E$ & $37.7 \mathrm{GPa}$ \\
\hline$v$ & 0.2 \\
\hline$Y_{D 0}$ & $1.10^{-4}$ \\
\hline$A_{t}$ & 1.0 \\
\hline$B_{t}$ & 15,600 \\
\hline
\end{tabular}

Table 15.1. Parameters of Mazars' damage model

The internal length $l_{c}$ is arbitrarily chosen equal to $0.02 \mathrm{~m}$, while the parameter $\lambda$, which influences the width of a damaged band, is chosen equal to 2 . The initial permeability $k_{0}$ considered in simulations is taken equal to $10^{-17} \mathrm{~m}^{2}$ and $\zeta=1$.

Evolutions of permeability, according to the proposed matching laws (equations [15.15] and [15.16]) with damage and with state variable are shown in Figure 15.3. The matching law provides a correct representation of the permeability for small damage, as well as for intense damage where it tends towards the permeability given by Poiseuille's law. 


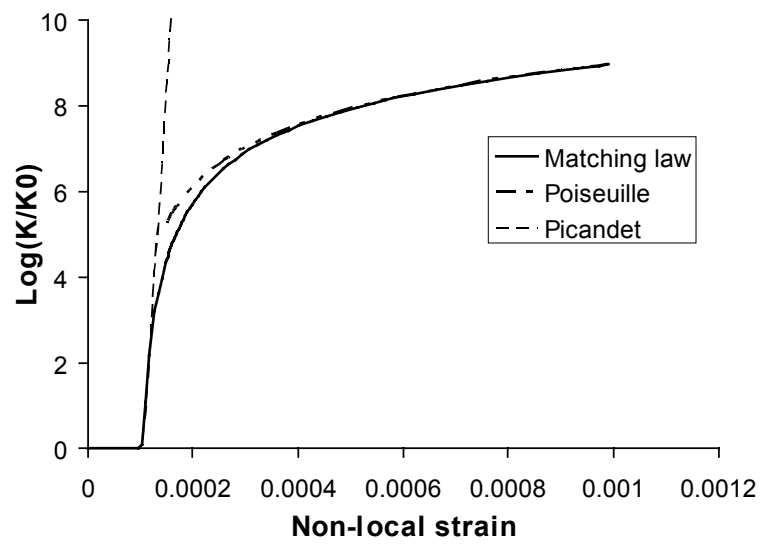

Figure 15.3. Logarithm of permeability evolution with state variable for the retained matching permeability law

\subsection{Structural simulations}

In order to illustrate the influence of the matching permeability law on a structural test, we consider concrete disks loaded either according to the splitting test configuration or to the "Bipede" configuration (Gérard, 1996). In this latter configuration, a disk made of mortar is glued to steel plates (Figure 15.4). Tension is applied to the plates and it is transmitted to the disk in which one or two cracks appear. At the same time, water flows through holes in the steel plates and through the disk. The apparent permeability (to water) is measured in the course of loading and cracking of the disk.

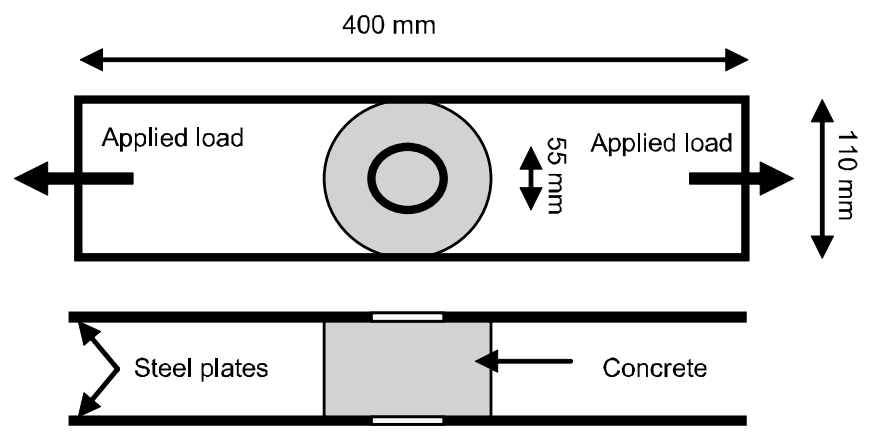

Figure 15.4. Bipede test geometry (after Gérard, 1996) 
In the present comparisons, we shall consider the tests in which a single crack developed. Note that in the splitting test and in the Bipede test, the two distributions of damage are quite similar.

\subsubsection{Mechanical problem - Brazilian splitting test}

The Brazilian splitting test is used as a standard measure of tensile strength of concrete, rocks and other geomaterials. The cylindrical specimen is loaded along a diametral plane by means of steel bearing plates, as shown in Figure 15.5(a). The steel bearing plates are arbitrarily modeled with rigid plates, with high Young's modulus $(E=300 \mathrm{GPa})$ and Poisson's ratio $v$ of concrete in order to avoid a confinement effect on concrete. The set of parameters of the integral non-local damage model (see Table 15.1) represents ordinary concrete behavior. The internal length is equal to $0.02 \mathrm{~m}$.

Numerical simulations are performed with 4-node quadrangle elements. Due to double symmetry, the computational domain consists of one quarter of a specimen. A plane stress calculation is carried out.

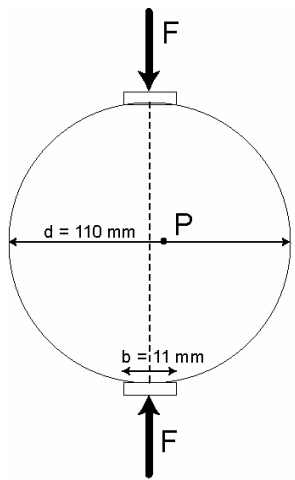

(a)

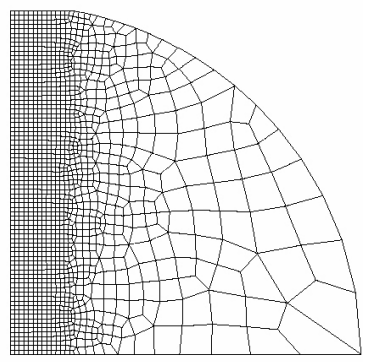

(b)

Figure 15.5. Brazilian splitting test: (a) problem statement; (b) FE mesh

This non-linear problem is solved incrementally by crack opening displacement (COD) control, i.e. the horizontal displacement of point $\mathrm{P}$. The plot of the applied load versus COD is shown in Figure 15.6. 


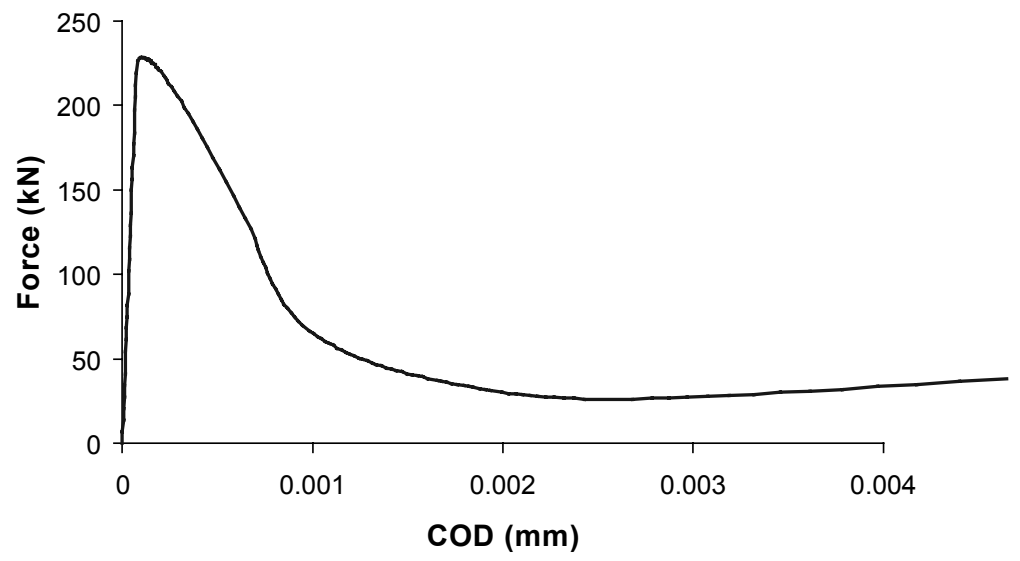

Figure 15.6. Force versus crack opening displacement in the Brazilian splitting test

Damage distributions at peak and at the last loading step are depicted in Figures 15.7(a) and (b). Maximal damage is initially located in some places along the vertical symmetry axis and then it translates downwards to the center as the loading increases. We can observe that damage develops in a band of a limited width (governed by the internal length). In addition, the height of the damage band at failure is the diameter.

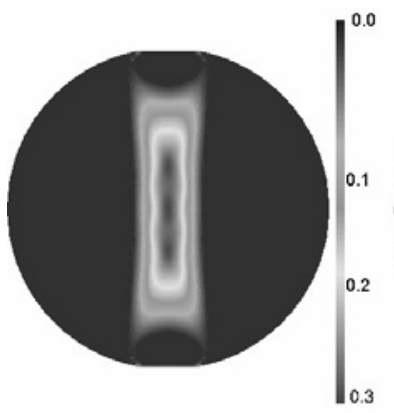

(a)

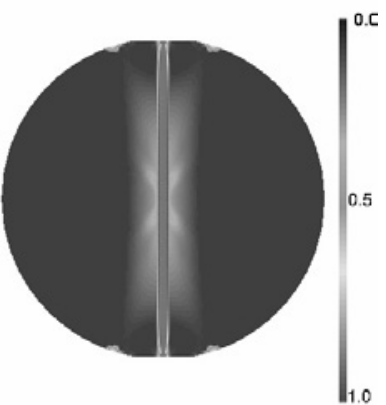

(b)

Figure 15.7. Damage distributions at (a) peak and (b) at the last loading step 


\subsubsection{Evolution of apparent permeability}

For each damage state, a local permeability is calculated according to the matching law at each Gauss point of a discretised structure. The expression of the crack opening displacement is taken from equation [15.20] under the assumption that strain and damage are constant within the damage band. This is not exactly true. It requires that the crack band width be small compared to the size of the structure so that variations within the band may be neglected. Then, a structural permeability is determined by a standard averaging of the local permeabilities. Results are plotted in Figure 15.8 which shows the evolution of the average permeability with the COD to peak COD ratio. We can observe that the shape of the curve is similar to that in Figure 15.1. Initially, the permeability increases very quickly and at failure the rate of growth decreases. The first regime covers a small range but the two subsequent regimes are quite well represented.

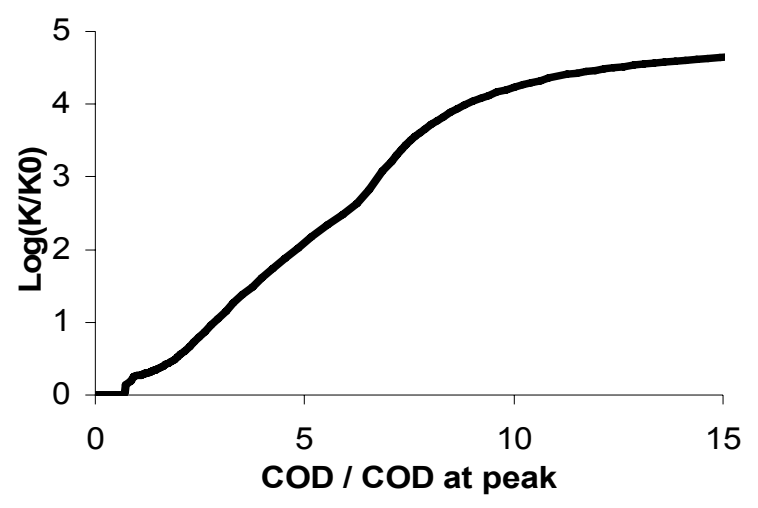

Figure 15.8. Evolution of logarithm of structural relative permeability with $C O D$ to peak $C O D$ ratio

Quantitative comparisons with experiments can be provided from the data obtained in the "Bipede" configuration. We consider first the results reported by Jason et al. (2007). In Figure 15.9, the comparison between the predictions of average permeability calculated by considering that locally the permeability follows the diffuse damage equation (Picandet's relationship) and the test data is presented. This comparison is very weak, which justifies the need for a permeability law that matches properly the two extreme cases of diffuse and localized damage. The second one cannot be obtained as a consequence of localization of damage, with the permeability law corresponding locally to diffuse damage. 


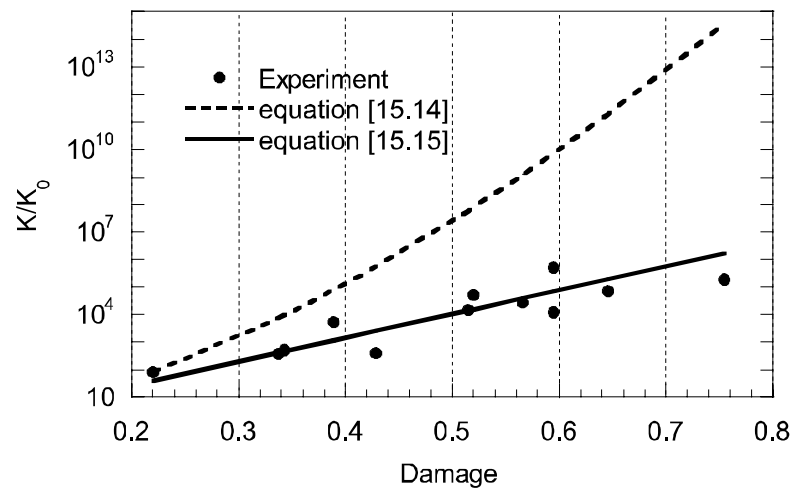

Figure 15.9. Evolution of the permeability with damage. Comparison of Picandet's relationship (denoted as equation [15.14]) and a power law for damage (denoted as equation [15.15]) with experimental data

It is not possible to capture the evolution of the apparent permeability with the diffuse damage case alone. This relationship overestimates the variation of permeability locally and it is not because such an overestimation occurs in a very small region of the structure (damage localization band) that it can be neglected. Figure 15.10 shows the comparison between the same test data and the present matching law. In the comparison, we have entered in our formula the same physical constants as those in Gérard (1996): the parameters of the damage model, the crack roughness and the dynamic viscosity of water. We have kept the same coordinate system which relates the applied strain to the water permeability of the cracked specimens.

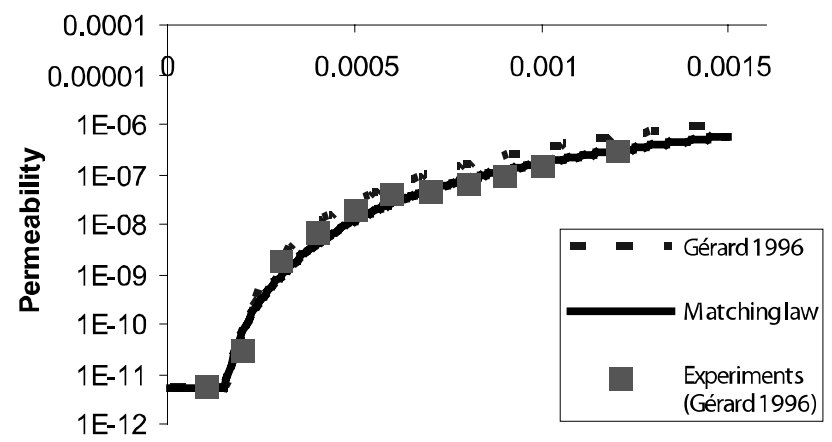

Deformation

Figure 15.10. Evolution of the permeability to water with the strain in the "Bipede" test 


\subsection{Conclusions}

In this contribution, a formula describing the evolution of permeability with damage has been proposed. It matches consistently two extreme cases: in the first case, permeability is an exponential function of distributed damage, while in the second case it is governed by crack opening. By analytical variable substitution we have associated the crack opening with the variable that governs damage in a nonlocal integral damage model and afterwards we have related this state variable with damage in order to arrive at an expression where the permeability is controlled solely by the variation of material damage.

An extensive comparison of the predictions of permeability with experimental data on split cylinders is in progress, but initial comparisons provide qualitative and quantitative results that are quite consistent.

\subsection{Acknowledgement}

Financial support from the Agence Nationale de la Recherche under the project "Contifiss" is gratefully acknowledged.

\subsection{Bibliography}

Bazant Z.P. and Planas J. (1998), Fracture and Size Effect in Concrete and Other Quasibrittle Materials, CRC Press.

Chatzigeorgiou G., Picandet V., Khelidj A. and Pijaudier-Cabot G. (2005), "Coupling between progressive damage and permeability of concrete: analysis with a discrete model", Int. J. Anal. Meth. Geomech., 29: 1005-1018.

Choinska M., Khelidj A., Chatzigeorgiou G. and Pijaudier-Cabot G. (2007), "Effects and interactions of temperature and stress-level related damage on permeability of concrete", Cement and Concrete Research, 37: 79-88.

Dormieux L. and Kondo D. (2004), "Approche micromécanique du couplage perméabilitéendommagement", C.R. Mecanique, 332: 135-140 (in French).

Dufour F., Pijaudier-Cabot G., Choinska M. and Huerta A. (2008), "Extraction of crack opening from a continuous approach using regularised damage models", Computers and Concrete, submitted.

Gérard B. (1996), Contribution of the mechanical, chemical, and transport couplings in the long-term behavior of radioactive waste repository structures. PhD Dissertation, Univ. Laval and Ecole normale supérieure de Cachan (in French).

Jason L., Ghavamian S., Pijaudier-Cabot G. and Huerta A. (2004), "Benchmarks for the validation of a non-local damage model", Revue Française de Génie Civil, 8: 303-328. 
Jason L., Pijaudier-Cabot G., Ghavamian S. and Huerta A. (2007), "Hydraulic behaviour of a representative structural volume for containment buildings", Nuclear Engrg. and Design, 237:1259-1274.

Mazars J. and Pijaudier-Cabot G. (1996), "From damage to fracture mechanics and conversely: a combined approach”, Int. J. of Solids and Structures, 33: 3327-3342.

Mazars, J. (1984), Application de la mécanique de l'endommagement au comportement non linéaire et à la rupture de béton de structure. PhD Dissertation, Pierre and Marie Curie University.

Picandet V., Khelidj A. and Bastian G. (2001), "Effect of axial compressive damage on gas permeability of ordinary and high-performance concrete", Cement and Concrete Research, 31: 1525-1532.

Pijaudier-Cabot G. and Bazant Z.P. (1987), "Nonlocal Damage Theory", Journal of Engineering Mechanics, 113: 1512-1533. 